\title{
Validity of Acute Cardiovascular Outcome Diagnoses Recorded in European Electronic Health Records: A Systematic Review
}

This article was published in the following Dove Press journal: Clinical Epidemiology

\author{
Jennifer Davidson (1D' \\ Amitava Banerjee ${ }^{2}$ \\ Rutendo Muzambi (D) \\ Liam Smeeth' \\ Charlotte Warren-Gash' \\ 'Faculty of Epidemiology \& Population \\ Health, London School of Hygiene and \\ Tropical Medicine, London, UK; ${ }^{2}$ Institute \\ of Health Informatics, University College \\ London, London, UK
}

Background: Electronic health records are widely used in cardiovascular disease research. We appraised the validity of stroke, acute coronary syndrome and heart failure diagnoses in studies conducted using European electronic health records.

Methods: Using a prespecified strategy, we systematically searched seven databases from dates of inception to April 2019. Two reviewers independently completed study selection, followed by partial parallel data extraction and risk of bias assessment. Sensitivity, specificity, positive predictive value (PPV), and negative predictive value estimates were narratively synthesized and heterogeneity between sensitivity and PPV estimates were assessed using $\mathrm{I}^{2}$.

Results: We identified 81 studies, of which 20 validated heart failure diagnoses, 31 validated acute coronary syndrome diagnoses with 29 specifically recording estimates for myocardial infarction, and 41 validated stroke diagnoses. Few studies reported specificity or negative predictive value estimates. Sensitivity was $\leq 66 \%$ in all but one heart failure study, $\geq 80 \%$ for $91 \%$ of myocardial infarction studies, and $\geq 70 \%$ for $73 \%$ of stroke studies. PPV was $\geq 80 \%$ in $74 \%$ of heart failure, $88 \%$ of myocardial infarction, and $70 \%$ of stroke studies. PPV by stroke subtype was variable, at $\geq 80 \%$ for $80 \%$ of ischaemic stroke but only $44 \%$ of haemorrhagic stroke. There was considerable heterogeneity $\left(\mathrm{I}^{2}>75 \%\right)$ between sensitivity and PPV estimates for all diagnoses.

Conclusion: Overall, European electronic health record stroke, acute coronary syndrome and heart failure diagnoses are accurate for use in research, although validity estimates for heart failure and individual stroke subtypes were lower. Where possible, researchers should validate data before use or carefully interpret the results of previous validation studies for their own study purposes.

Keywords: validation, myocardial infarction, heart failure, stroke; routinely collected health data

\section{Introduction}

Ischaemic heart disease and cerebrovascular disease have been the leading causes of death globally for more than 15 years. ${ }^{1}$ In Europe, cardiovascular disease (CVD) deaths and prevalence have decreased but remain substantial; in 2015 an estimated 85 million people had CVD including 11.3 million with new diagnoses. ${ }^{2}$

CVD determinants and outcomes research increasingly utilize electronic health records (EHRs). EHRs contain comprehensive longitudinal health data, extracted from primary and secondary care clinical systems, for large patient populations which provide cost-effective data for research. EHR data is mostly "structured"

Correspondence: Jennifer Davidson Email Jennifer.Davidson@Ishtm.ac.uk
Clinical Epidemiology 2020:12 1095-IIII 
with diagnoses coded using, for example, the International Classification of Diseases (ICD) but can also be "unstructured" with anonymized free-text notes. ${ }^{3}$ EHR-based research predominantly uses structured data. As the primary purpose of EHR data collection is clinical, it is essential to consider the validity of the data's use in research.

EHR use is widespread in Europe, where many countries have national healthcare systems, and several systematic reviews have previously explored the quality of specific European EHRs. ${ }^{4-7}$ Other systematic reviews ${ }^{8-12}$ have investigated the validity of CVD diagnoses in computerized health-related records, which included EHRs but mainly drew results from disparate claims-based systems. The previous reviews did not separate results for EHR and claims data, the quality of which may differ due to the differences in setup and collection rationale.

In our systematic review, we provide an up-to-date assessment of the validity of acute CVD diagnoses recorded in European EHRs. We defined acute CVD as heart failure (HF), acute coronary syndrome (ACS), and stroke. These high-burden conditions are key diagnoses commonly included in the composite endpoint of major adverse cardiovascular events (MACE) which is increasingly employed in both clinical trials and observational research studies. ${ }^{13}$ We investigated whether the validity of these diagnoses differed by subtype, definition, data source, reference standard, and study population.

\section{Methods}

\section{Protocol and Registration}

Our protocol was published in October $2019^{14}$ following the Preferred Reporting Items for Systematic Reviews and Meta-Analyses Protocol guidelines (PROSPERO registration number CRD42019123898).

\section{Eligibility Criteria}

We included articles that validated diagnoses in patients aged $\geq 16$ years captured in any European primary or secondary care EHR. We excluded claims-based databases, disease registries, vital registration systems, or locally held databases. Articles needed to validate clinical codes for the diagnoses of HF, ACS, or stroke (Table 1) against a suitable internal or external reference standard. $\mathrm{HF}$ is most frequently a chronic condition which can deteriorate with acute exacerbations. HF may also have an acute onset, for example after an MI. The European Society of Cardiology (ESC) defines acute HF as rapid onset or worsening of symptoms and/or signs of existing HF. ${ }^{15}$ ACS encompasses different clinical forms of myocardial ischaemia which includes myocardial infarction (MI) and unstable angina. The specific diagnosis of MI or unstable angina depends on symptoms, signs, biomarkers, and ECG and/or autopsy findings, with the definitions refined over time. ${ }^{16}$ The diagnosis of stroke includes subtypes ischaemic stroke, intracerebral haemorrhage (ICH), and subarachnoid haemorrhage (SAH). ${ }^{17}$ At least one validation estimate (Figure 1) or the raw data to calculate it was required.

\section{Information Sources}

We searched for eligible articles in five databases (Medline, Embase, Scopus, Web of Science, and Cochrane Library), two grey literature sources (OpenGrey and Ethos), and, where available, the bibliographies of EHR databases from the date of inception to April 2019 in any language.

\section{Search Strategy}

We searched medical subject heading terms and free-text (in the title and abstract) for the concepts of (1) CVD

Table I Example Clinical Codes Included for Stroke, Acute Coronary Syndrome and Heart Failure Diagnosis Definitions

\begin{tabular}{|l|l|l|l|l|}
\hline Diagnosis & Subtype & ICD-10 & ICD-9 & ICPC \\
\hline Acute coronary syndrome & Myocardial infarction & 410 & \\
& Unstable angina & 121 & 41 \\
& Cardiac arrest & 120.0 & K75 \\
& Other acute heart disease & 146 & 428 & \\
\hline Heart failure & & 124 & 430 \\
Stroke & Subarachnoid haemorrhage & 160 & 431,432 \\
& Intracerebral haemorrhage & 161 & 433,434 \\
& Cerebral infarction & 163 & 436 \\
\hline
\end{tabular}




\section{Reference standard}

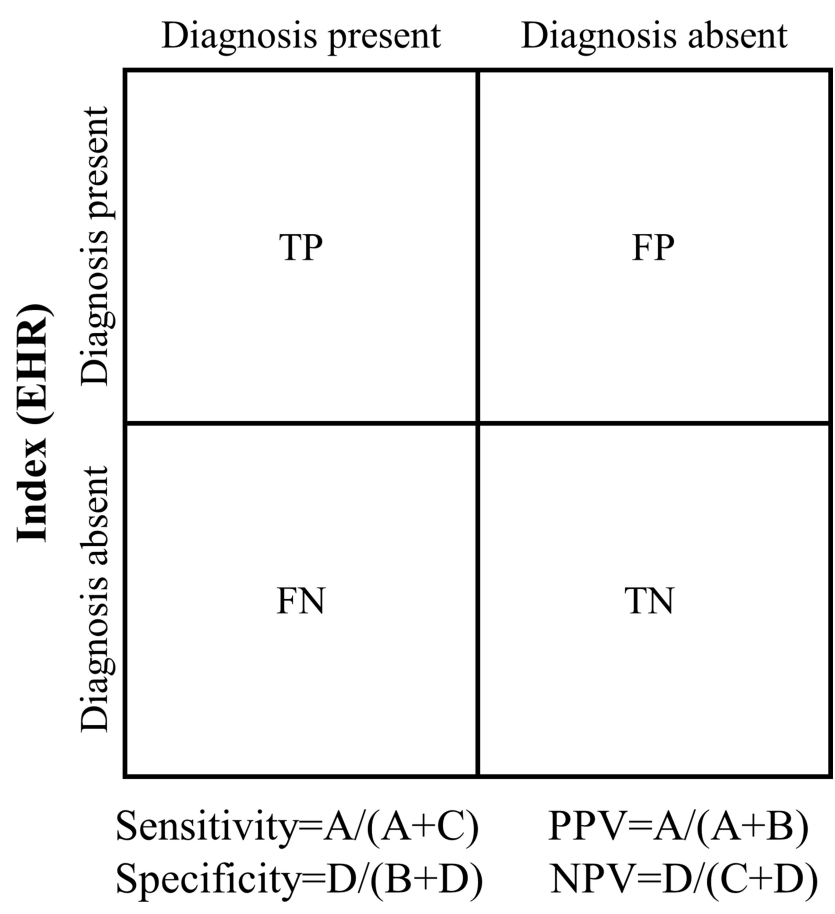

Figure I Illustration of validity estimates calculations.

diagnoses, (2) EHRs, (3) Europe, and (4) validation. Search terms were developed for Medline and transcribed for the remaining databases (S1 Appendix). To identify any additional articles, we checked reference lists of eligible articles and relevant systematic reviews.

\section{Study Selection and Data Collection}

Two reviewers (J.A.D. and R.M.) independently screened the titles and abstracts of all retrieved articles, followed by the full-text of articles deemed eligible in the first stage. Our published protocol details the full data collection process. ${ }^{14}$ Briefly, we extracted data using a pre-defined template (S2 Appendix) which we piloted using dual extraction for three studies, followed by further parallel extraction for $20 \%$ of studies, and completed by a single reviewer (J.A.D.) for the remaining studies.

\section{Risk of Bias in Individual Studies}

We used a modified version of the Quality Assessment of Diagnostic Accuracy Studies 2 (QUADAS-2) ${ }^{18}$ tool to assess bias (S3 Appendix). As with our data extraction, two authors (J.A.D. and R.M.) piloted the tool for three studies, then independently assessed risk in a further $10 \%$ of studies, with the process completed by a single reviewer (J.A.D.).

\section{Synthesis of Results}

We synthesized results with a narrative approach, grouping studies by acute CVD diagnosis (HF, ACS or stroke) and, where possible, subgroups of interest. Subgroups were; diagnosis type, definition, data source including diagnostic position and coding system, reference standard, and study population including time period, age and sex. For studies that reported validation estimates without confidence intervals (CIs), but included raw data, we calculated 95\% CIs using the Wilson method for binomial proportions. We used the $\mathrm{I}^{2}$ statistic to assess heterogeneity between the sensitivity and positive predictive value (PPV) estimates, following the Cochrane thresholds. ${ }^{19}$ Heterogeneity assessment did not include specificity or negative predictive value (NPV), as few studies reported these measures. To investigate sources of heterogeneity, we compared $\mathrm{I}^{2}$ before and after removing studies at a high risk of bias and by the previously mentioned subgroups. We used the Stata metaprop command ${ }^{20}$ to calculate $\mathrm{I}^{2}$. Metaprop uses raw data rather than precalculated estimates; studies that reported sensitivity or PPV but not the data used to calculate were excluded from heterogeneity assessment.

\section{Risk of Bias Across Studies}

We used the Grading of Recommendations, Assessment, Development, and Evaluation (GRADE) tool for diagnostic accuracy systematic reviews ${ }^{21}$ to summarise crossstudy quality. Evidence was categorised as "high", "moderate", "low" or "very low" quality. See S4 Appendix for the reasons we rated quality down or up.

\section{Results}

\section{Studies Included}

We identified 4595 studies, of which 218 were included in full-text review and 81 met eligibility criteria (Figure 2).

Study characteristics are summarized in S1 Table, results are displayed in $\underline{\mathrm{S} 2}$ Table, Figures $3-8$ and $\underline{\mathrm{S} 1-6}$ $\underline{\text { Figs, }}$ additional subgroup results are described in $\underline{\mathrm{S} 5}$ Appendix, QUADAS-2 results are in S3 Table, and our GRADE assessment is detailed in $\mathrm{S} 4$ Table.

\section{Study Characteristics}

The 81 included studies validated EHRs from 11 different countries, most frequently Denmark (18 studies) $)^{22-39}$ and the UK (17 studies). ${ }^{40-56}$ Validation was the primary aim of all but 10 studies. $^{35,36,41,48,57-62}$ Fourteen studies ${ }^{26,27,31,63-73}$ validated a vital registration system or disease registry in 


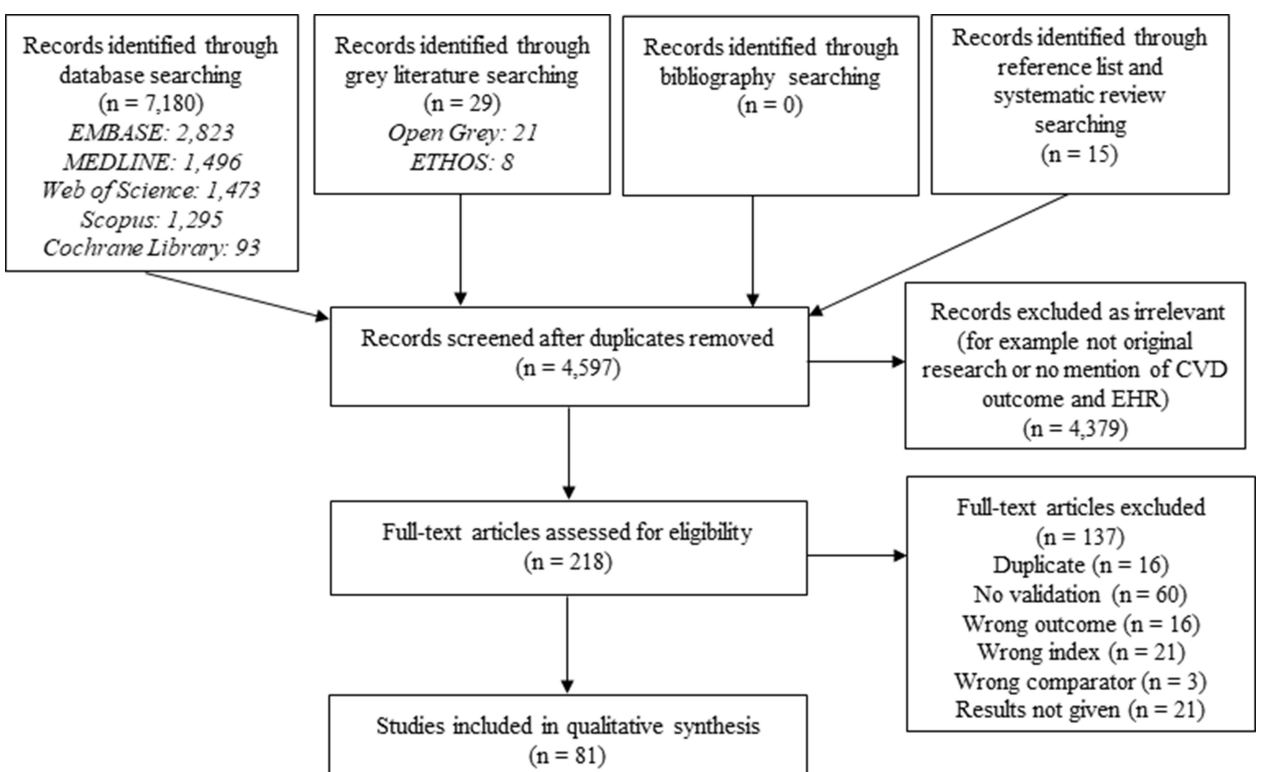

Figure 2 Flow diagram of study selection.

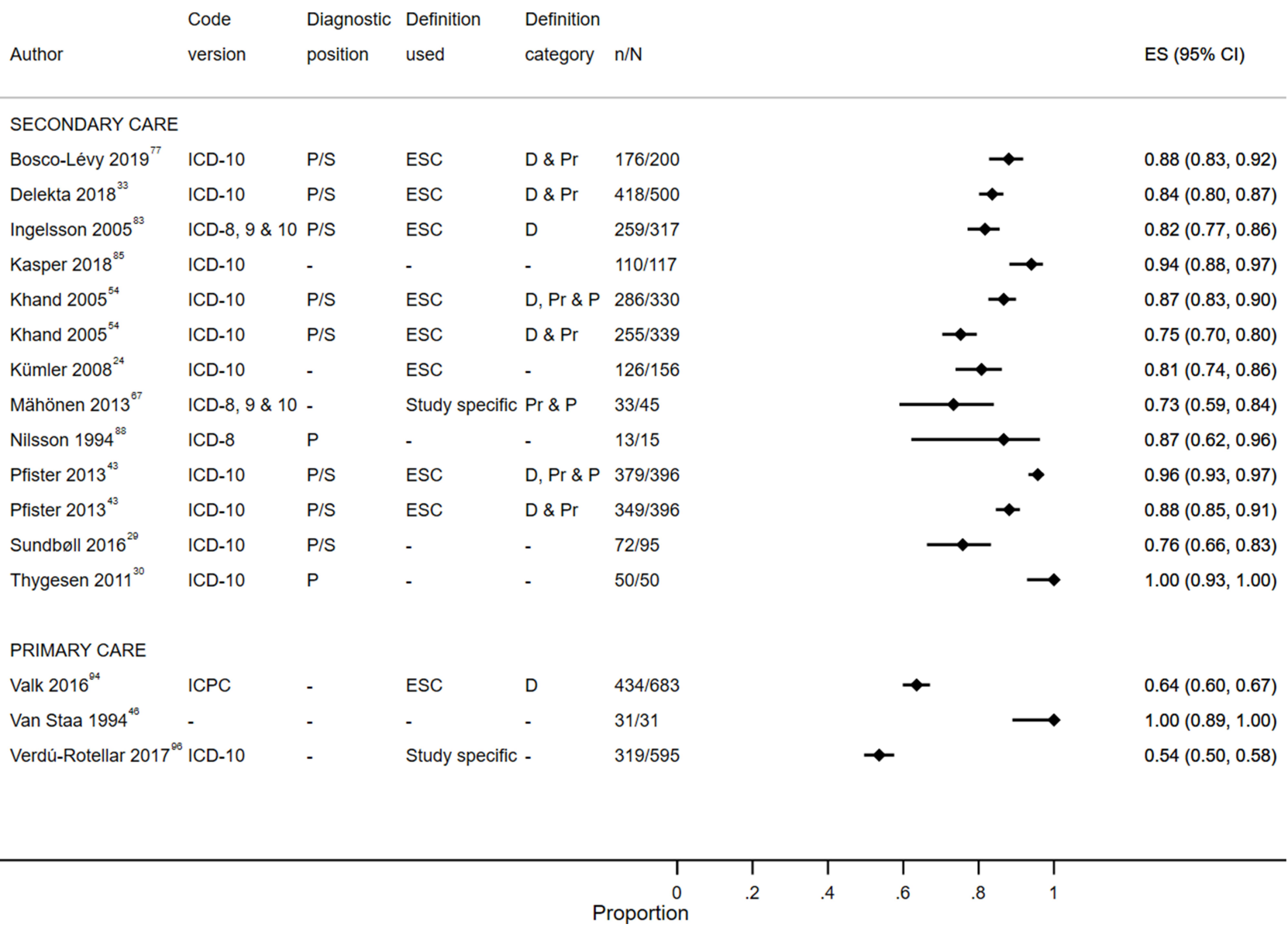

Figure 3 Positive predictive value for heart failure diagnoses from studies which reported the number of records confirmed positive and the total number of records. Abbreviations: D, definite; D \& Pr, definite and probable; $D, \operatorname{Pr} \& \mathrm{P}$, definite, probable and possible; $\mathrm{P}$, primary; $\mathrm{P} / \mathrm{S}$, primary or secondary. 


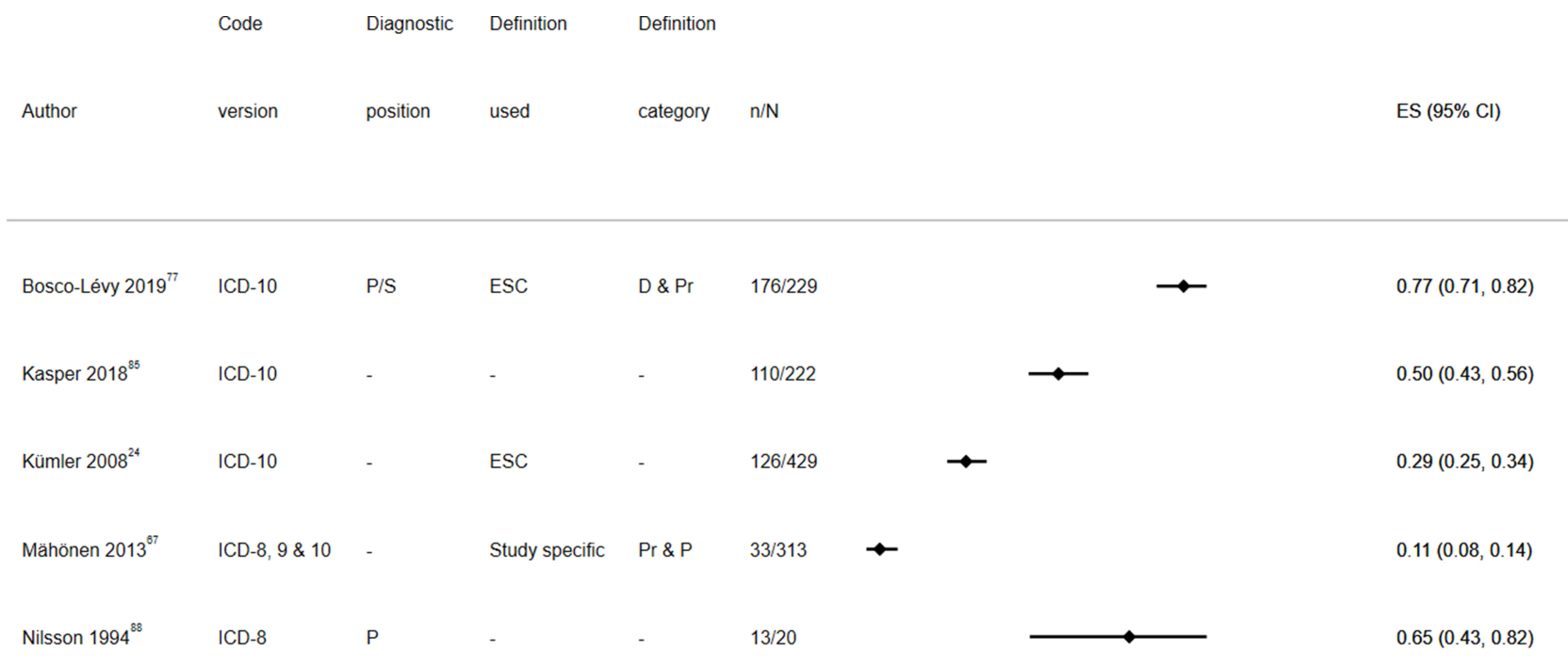

$\begin{array}{rrrrrr}1 & 1 & 1 & 1 & 1 & 1 \\ 0 & 2 & 4 & .6 & .8 & 1 \\ \text { Proportion } & & & & \end{array}$

Figure 4 Sensitivity for heart failure diagnoses from studies which reported the number of records confirmed positive and the total number of records. Abbreviations: D \& Pr, definite and probable; Pr \& P, probable and possible; P, primary; P/S, primary or secondary.

addition to the EHR. The records validated included data from 1969-2015. Where ICD coding was validated this covered versions 8-10. Sixty studies used medical record review as a reference standard. 22,23,25-39,42,43,45,46,49, 50,54,55,57-63,69,72,74-96 Twenty studies validated $\mathrm{HF}^{24,28-}$ $30,33,43,46,54,59,65,67,77,82,83,85,88,94-97 \quad 31 \quad \mathrm{ACS}^{22,23,26,27,29,30}$ $32,34,37,42,46,47,50,52,53,58,65,68-70,75,76,80,81,84,87,88,91,98-100$ and 41 stroke diagnoses. $25,31,32,35,36,38-41,44,45,47-49,51,55-57,60-64$, 66,71-74,78,79,81,86,87,89-93,98,101,102

\section{Study Quality}

Study quality was high for $54(67 \%)$ of studies, ${ }^{22-}$ $26,28,29,31-34,38,39,42-44,47,50,51,53,54,56,59,60,62-65,67-70,72$,

73,75-79,85-90,92-94,96,98-102 medium for 19 (24\%) studies $^{27,30,35-37,46,49,52,55,57,58,61,66,74,81-84,95}$ and low for eight $(10 \%)$ of studies. $40,41,45,48,71,80,91,97$ Studies were overall at low risk of bias in patient selection (76 low, 3 unclear, 2 high), index test (71 low, 10 high), and flow and timing (78 low, 3 unclear) domains and higher risk in the reference standard domain (36 low, 28 unclear, 17 high). Generally, reference standard methods and definitions were poorly described, and on occasion the reference standard was not independent of the EHR. Risk of bias was also higher in studies which validated primary care EHRs. HF validation studies had high quality in $14(70 \%)$ studies, medium in five $(25 \%)$ and low in one $(5 \%)$. For ACS validation, quality was high for $21(68 \%)$, medium for eight (26\%) and low for two $(6 \%)$ studies. In stroke validation studies, quality was high for $26(63 \%)$, medium for nine $(22 \%)$ and low for six (15\%) studies.

\section{Heart Failure Study Characteristics}

HF diagnoses were most extensively validated using EHR data from Denmark (five studies), ${ }^{24,28-30,33}$ the Netherlands (four studies), ${ }^{59,65,94,95}$ Sweden (three studies) ${ }^{82,83,88}$ and the UK (three studies). ${ }^{43,46,54}$ In addition, EHR data from Finland, ${ }^{67}$ France, ${ }^{77}$ Germany, ${ }^{85}$ Italy $^{97}$ and Spain ${ }^{96}$ were validated in one study each. Fourteen studies validated secondary care $\mathrm{EHRs}^{24,28-30,33,43,54,59,65,67,77,83,85,88}$ and six studies validated primary care EHRs. ${ }^{46,82,94-97}$ Medical record review was used as the reference standard in all but three studies. ${ }^{24,65,97}$

\section{Heart Failure Validation Results Overall}

From the main validation result reported by each of the studies; sensitivity (available from nine studies)$24,46,65,67,77,82,85,88,95$ was $\geq 50 \%$ in six studies $46,77,82,85,88,95$ but $>66 \%$ (range $11-100 \%$ ) in only one study, ${ }^{46}$ PPV (19 


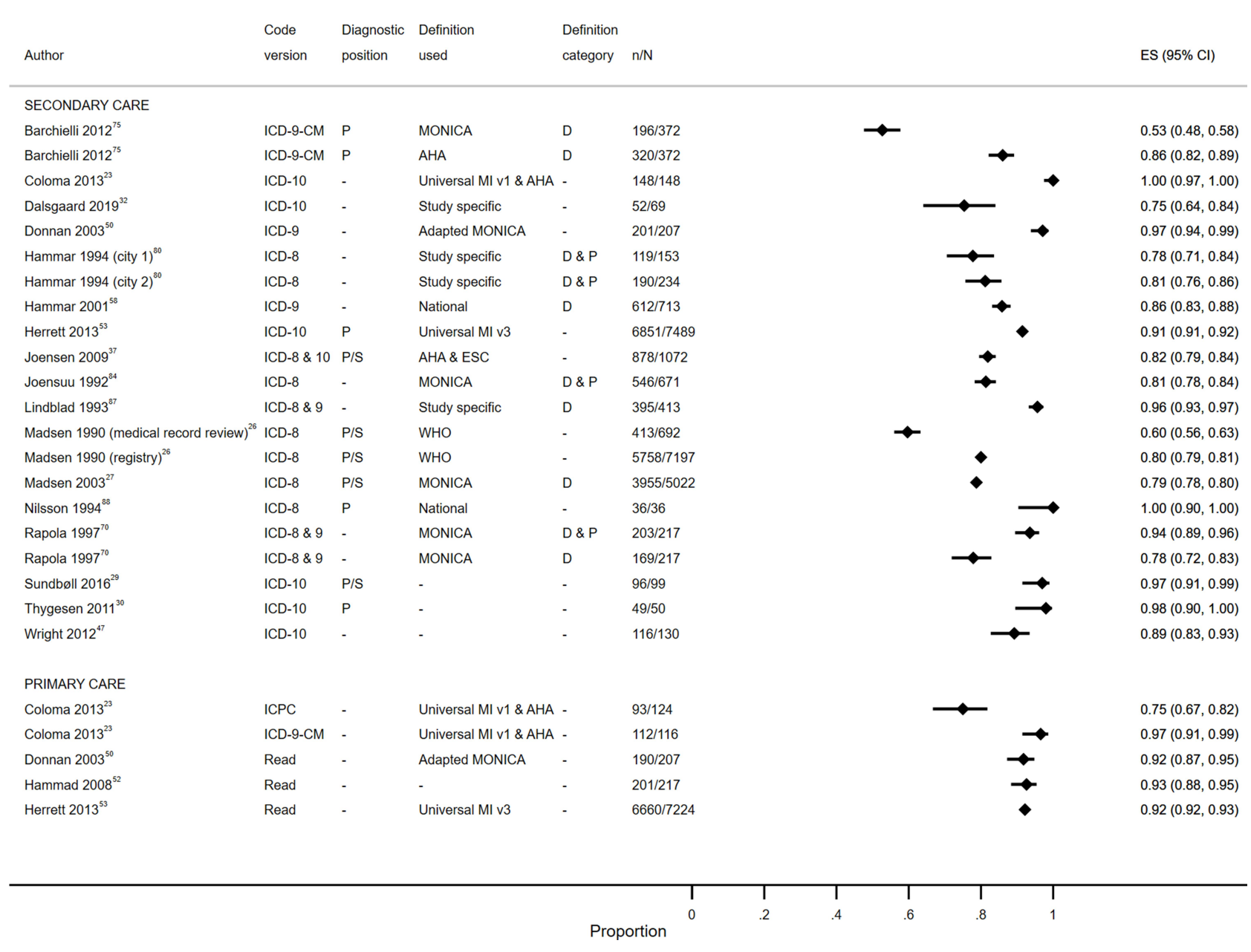

Figure 5 Positive predictive value for myocardial infarction diagnoses from studies which reported the number of records confirmed positive and the total number of records.

Abbreviations: D, definite; D \& P, definite and possible; P, primary; P/S, primary or secondary; RS, reference standard.

studies) $)^{24,28-30,33,43,46,54,59,65,67,77,83,85,88,94-97}$ was $\geq 80 \%$ (range 54-100\%) in all but five studies, ${ }^{29,67,94,96,97}$ specificity (three studies) ${ }^{24,67,95}$ was $\geq 95 \%$ in all studies, and NPV (three studies) (24,67,95 $^{2}$ was $\geq 84 \%$ (range $84-96 \%$ ) in all studies.

\section{Diagnosis Type}

In the three studies that reported results for first diagnosis, the PPV range was $76-88 \% .^{28,29,77}$ One study compared the PPV for all diagnoses (84\%) to first diagnosis $(80 \%),{ }^{28}$ and another study found the same PPV for first diagnosis and recurrent diagnosis (both $76 \%)^{29}$

\section{Definition}

In seven of the eight studies $24,28,33,43,54,77,83,94$ which used the ESC definition, ${ }^{15}$ the PPV was $\geq 80 \%$. The study ${ }^{94}$ with the lower PPV of $64 \%$ was the only one to validate a primary care EHR. Other studies used; both Framingham $^{103}$ and Boston ${ }^{104}$ criteria (one study, ${ }^{59}$ PPV
80-81\%), the American College of Cardiology (ACC)/ American Heart Association (AHA) definition ${ }^{105}$ (one study, ${ }^{97}$ PPV 55\%), or study-specific definitions (three studies, ${ }^{67,95,96}$ PPV 54-83\%). An overview of the definitions used by the studies is presented in S6 Appendix.

Seven studies reported classification criteria; the PPV for definite HF ranged between $61-82 \%, 33,43,54,77,83$ including both definite and probable HF increased the PPV to $73-88 \%^{33,43,54,77,83,94}$ and the two studies which additionally included possible HF reported high PPV as $87 \%{ }^{54}$ and $96 \%{ }^{43}$

\section{Diagnostic Position}

Six studies ${ }^{29,33,43,54,77,83}$ reported $\mathrm{HF}$ recorded in any diagnostic position (PPV 76-96\%) and two studies ${ }^{30,88}$ only included primary position (PPV 87\% and 100\%). Three studies, ${ }^{33,77,83}$ which validated any position, also included breakdowns by primary (PPV 88-96\%) and secondary (PPV 66-84\%) positions. 


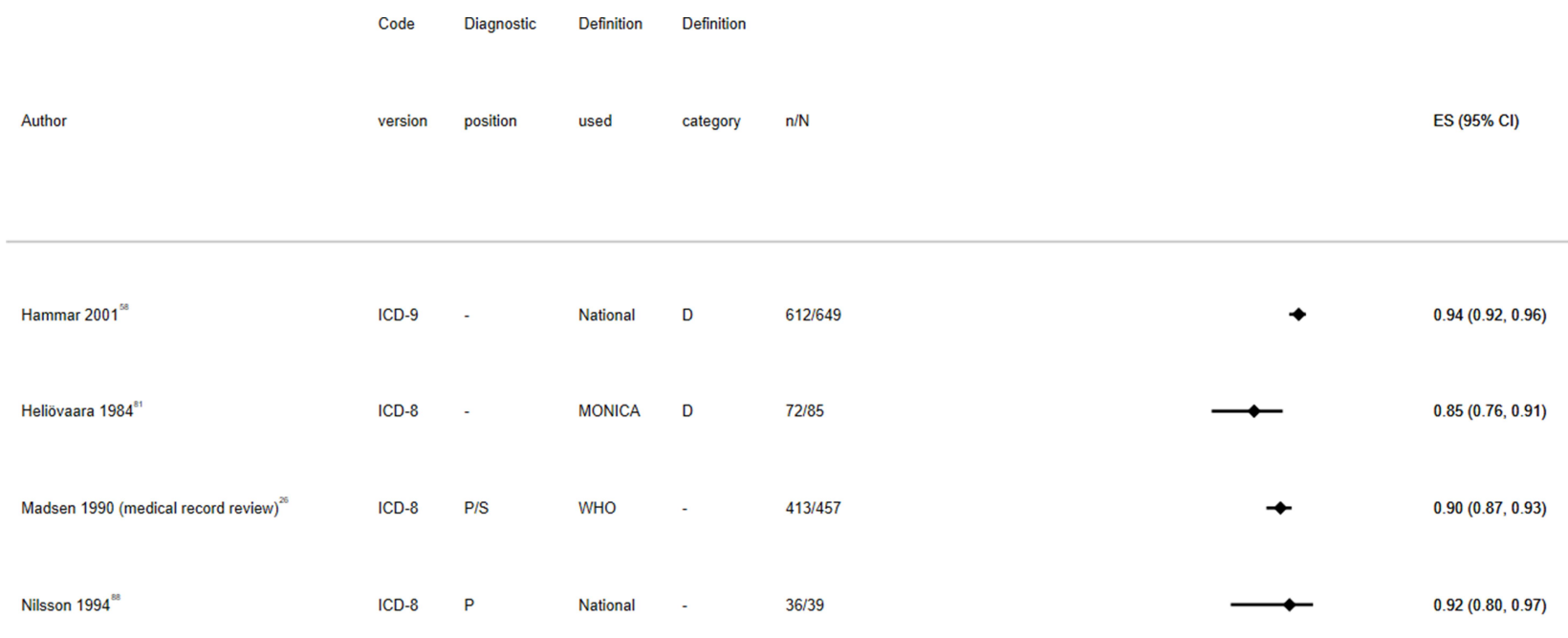

Proportion

Figure 6 Sensitivity for myocardial infarction diagnoses from studies which reported the number of records confirmed positive and the total number of records. Abbreviations: D, definite; D \& P, definite and possible; P, primary; P/S, primary or secondary; RS, reference standard.

\section{Coding System}

Twelve studies validated ICD-10, ${ }^{24,28-30,33,43,54,67,77,82,83,96}$ with all but one ${ }^{83}$ reporting results specifically for this version of ICD (PPV 78-99\%). Six studies ${ }^{24,33,43,77,82,96}$ validated I50; two studies of primary care EHRs reported lower validity estimates (PPV 54\% ${ }^{96}$ and sensitivity $\left.66 \%\right)^{82}$ compared to four studies of secondary care EHRs (PPV $81-96 \%,{ }^{24,33,43,77}$ and sensitivity $29 \%{ }^{24}$ and $\left.64 \%\right){ }^{77}$ Five studies included a broader range of ICD-10 codes, all of which differed. The estimates for ICD-10 codes were no higher than those for ICD-8 (PPV 87\%), ${ }^{67,83,88}$ ICD-9 (PPV 79-97\%), ${ }^{59,65,67,83}$ or combinations of the three ICD systems (PPV 73-82\%). ${ }^{67,83}$ Two studies validated ICPC K77 in primary care EHRs (PPV 64\% ${ }^{94}$ and $83 \%{ }^{95}$ ).

\section{Acute Coronary Syndrome Study Characteristics}

Similar to HF, ACS diagnoses were most frequently validated using EHR data from Denmark (nine studies),22,23,26,27,29,30,32,34,37 followed by Finland (seven studies), ${ }^{68-70,81,84,99,100}$ the UK (six studies) ${ }^{42,46,47,50,52,53}$ and Sweden (4 studies). ${ }^{58,80,87,88}$ Two studies validated data in each of Italy, ${ }^{23,75}$ the Netherlands, ${ }^{23,65}$ and Spain, ${ }^{91,98}$ and a final study used data from France. ${ }^{76}$ Twenty-six of the studies validated a secondary care EHR, ${ }^{22,26,27,29,30,32,34,37,42,47,58,65,68-70,75,76,80,81,84,87,88,91, ~}$
98-100 three studies validated both a primary and secondary care $\mathrm{EHR}^{23,50,53}$ and two studies validated a primary care EHR. $^{46,52}$

Four studies ${ }^{22,37,68,76}$ presented overall ACS results, of which one study ${ }^{68}$ included an additional breakdown for MI and two studies ${ }^{37,76}$ included unstable angina and MI, one of which also included cardiac arrest. ${ }^{37} \mathrm{~A}$ further two studies $^{29,65}$ did not report results for ACS overall but did include both unstable angina and MI. The remaining 25 studies solely validated MI diagnoses. ${ }^{23,26,27,30,32 \text {, }}$ $34,42,46,47,50,52,53,58,69,70,75,80,81,84,87,88,91,98-100$

\section{Acute Coronary Syndrome Validation Results Overall}

For ACS, three studies $33,37,76$ reported one main PPV (range 66-87\%), while results presented by Pajunen et al ${ }^{68}$ were broken down by age, sex and time period, with sensitivity of $66-87 \%$ and PPV of $63-86 \%$.

\section{Diagnosis Type}

The PPV for unstable angina varied; with low values of $20 \%{ }^{76}$ and $27.5 \%{ }^{37}$ in two studies and higher values of $78 \%{ }^{65}$ and $88 \%{ }^{29}$ in the other two studies. Sensitivity was only reported by one study, ${ }^{65}$ at $53 \%$. For MI, the main validation result for sensitivity (11 studies) $26,27,34,42,46,50,58,65,81,88,98$ was $\geq 80 \%$ in 


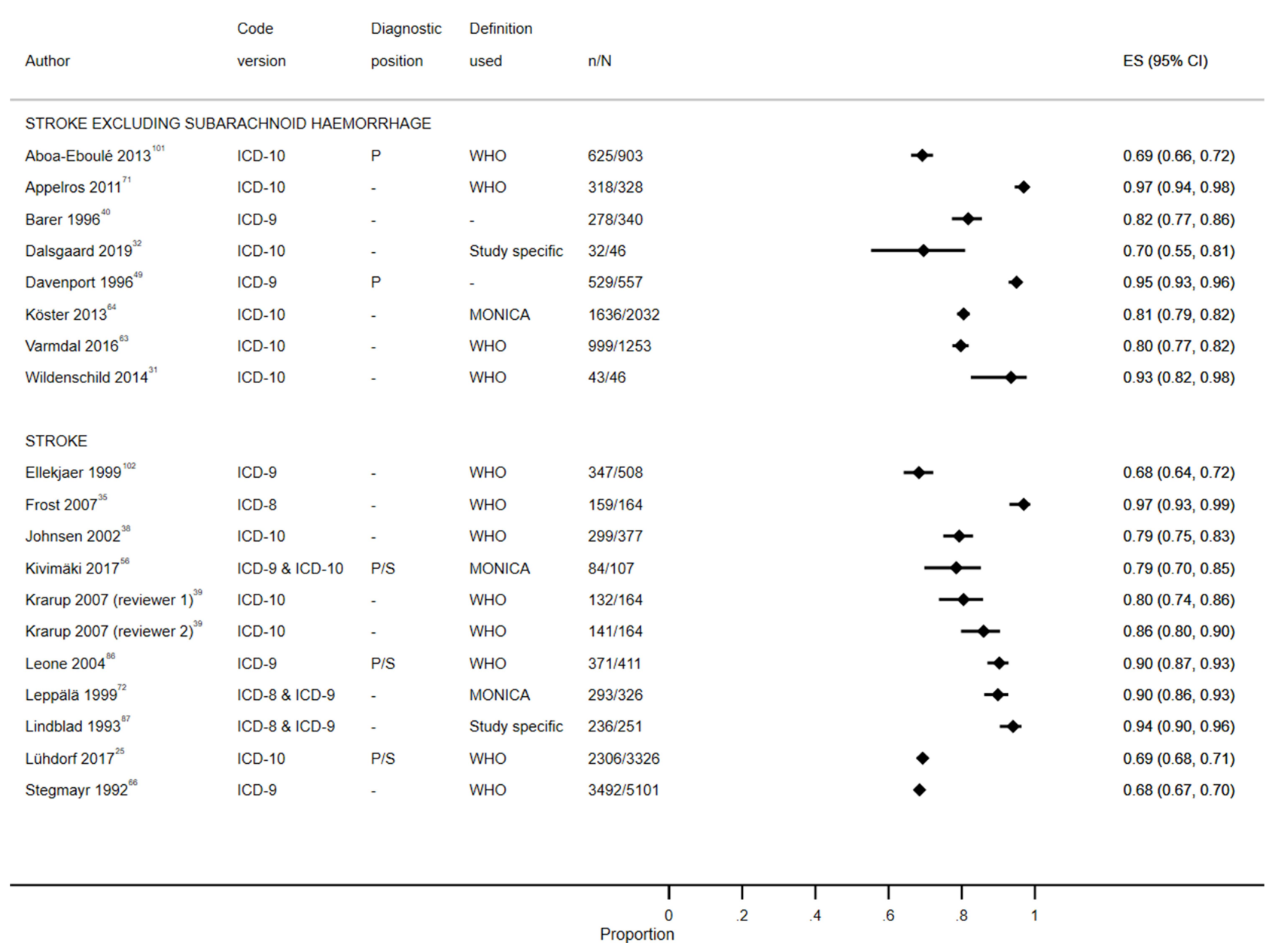

Figure 7 Positive predictive value for stroke diagnoses recorded in secondary care EHRs from studies which reported the number of records confirmed positive and the total number of records.

Abbreviations: ES, effect size; P, primary; P/S, primary or secondary.

all but one study ${ }^{42}$ (range 56-97\%), and $\operatorname{six}^{26,27,34,58,88,98}$

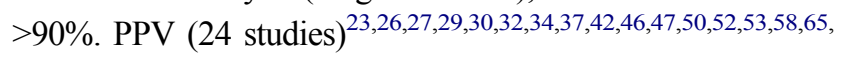
$70,75,76,80,84,87,88,98$ was $\geq 80 \%$ (range $42-100 \%$ ) in all but three studies $^{27,32,34}$ with $12^{23,29,30,42,50,52,53,65,87,88,98} \geq 90 \%$. Three studies $^{34,42,98}$ reported specificity (range 93-100\%) and two34,98 included NPV (range 82-100\%).

Four studies $^{29,32,37,84}$ reported the PPV for first MI, with estimates of 75-97\%, and one study ${ }^{29}$ also included recurrent MI with a PPV of $88 \%$ compared to $97 \%$ for first MI.

\section{Definition}

Varying MI definitions were used (S6 Appendix). Most frequently (nine studies) $26,27,50,70,75,81,84,99,100$ the World Health Organization (WHO) Monitoring trends and determinants in cardiovascular disease (MONICA) definition ${ }^{106}$ was used, with variable PPV estimates of 53-96\% obtained. Two studies compared MONICA to another MI definition; one ${ }^{75}$ showed MONICA-defined definite MI had a substantially lower PPV than AHA/ESC-defined ${ }^{16}$ definite MI $(53 \%$ vs
$86 \%$, while the other ${ }^{84}$ also showed a lower PPV for MONICA compared to "normal clinically defined MI" but with a smaller difference ( $81 \%$ vs $89 \%$ ). One further study used the AHA/ESC definition ${ }^{37}$ (PPV 82\%). The universal definition ${ }^{107}$ was used in a study ${ }^{23}$ which included EHR data from three countries, with PPVs of $75-100 \%$. Three studies used the third universal definition, ${ }^{108}$ one $^{76}$ of which combined it with the earlier universal definition (PPV 85\%). In another ${ }^{53}$ PPVs of $92 \%$ with obtained for the primary and secondary care EHRs validated. The third ${ }^{34}$ validated MI diagnoses recorded for patients with drug-eluting coronary stents, the PPV was $42 \%$ for all admission and $73 \%$ for acute admissions.

\section{Diagnostic Position}

Of the 10 studies which reported the diagnostic position used to validate MI diagnoses, five $\mathrm{e}^{26,27,29,34,68}$ used any diagnostic position (PPV 42-97\%) and five $\mathrm{e}^{30,75,76,88,98}$ primary position (PPV 53-100\%). One study ${ }^{27}$ which 


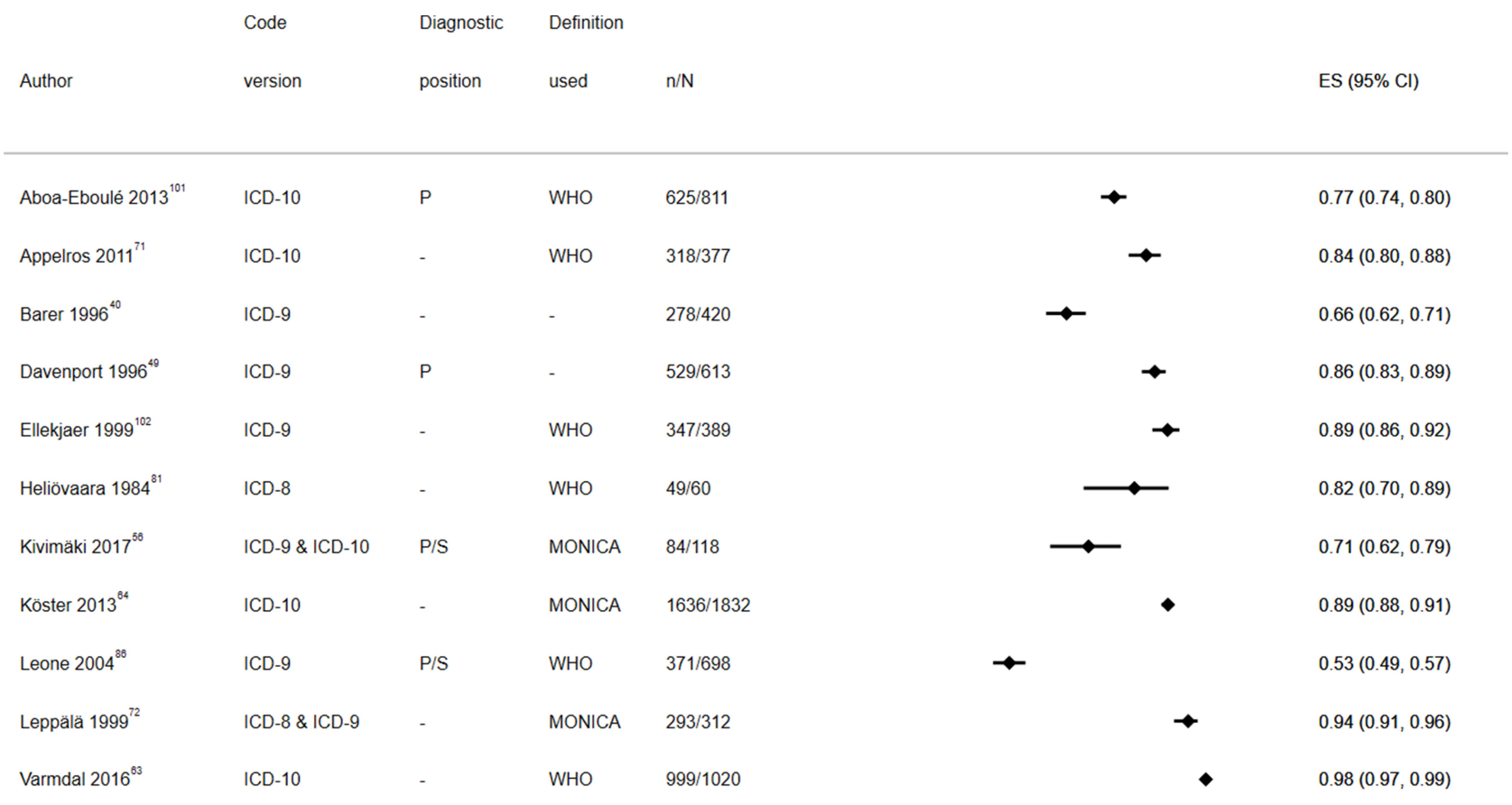

\begin{tabular}{rl|l|l|l|l|l}
\hline & 1 & 1 & 1 & 1 & 1 & 1 \\
Proportion & 2 & .4 & .6 & .8 & 1 & 1 \\
\hline
\end{tabular}

Figure 8 Sensitivity for stroke diagnoses recorded in secondary care EHRs from studies which reported the number of records confirmed positive and the total number of records.

Abbreviations: ES, effect size; P, primary; P/S, primary or secondary.

validated any position (PPV 79\%) also included a breakdown by primary position (PPV $80 \%$ ) and another study ${ }^{29}$ included breakdowns by primary (PPV 99\%) and secondary positions (PPV 80\%).

\section{Coding System}

Ten studies validated ICD-10 coded MI, eight reported results specifically for ICD-10. ${ }^{23,29,30,32,34,47,53,76}$ Four studies validated ICD-10 I21 with PPV $\geq 85 \%$ (range $42-100 \%)^{23,29,34,76}$ in all but one. ${ }^{34}$ Two studies included I21-I23 and reported high PPVs of $92 \%{ }^{53}$ and $98 \% ;^{30}$ however, the latter study was small in size (50 patients). One study validated I21-I22 (PPV 89\%) ${ }^{47}$ and another I21-I24 (PPV 75\%). ${ }^{32}$ The estimates for ICD-10 codes were no higher than those for ICD-8 (PPV $79-100 \%),{ }^{26,27,80,84,88}$ ICD-9 (86-100\%), ${ }^{42,50,58,65,75,98}$ or combinations of three ICD systems (PPV 82-96\%). ${ }^{37,87}$ Of the studies to validate data in primary care, one ${ }^{23}$ included IPCI K75 code (PPV 75\%) and three ${ }^{50,52,53}$ validated Read coding in the UK (PPV 91-93\%).

\section{Reference Standard}

The PPV for MI diagnoses varied between $53-100 \%$ when medical record review was the reference standard (20 studies) $)^{22,23,26,29,30,32,37,42,46,50,58,69,70,75,76,80,84,87,88,91}$ and $89-93 \%$ when a registry was used. ${ }^{26,27,53,68,98-100}$ One study $^{34}$ used medical record review after comparing EHR and registry results (PPV 42\%). Two studies used a GP questionnaire (PPV 89\% and 93\%), ${ }^{47,52}$ and one study used a local cardiology database (PPV 97\%). ${ }^{65}$

\section{Stroke Study Characteristics}

Stroke diagnoses were most frequently validated in UK EHRs, with 10 studies conducted, ${ }^{40,41,44,45,47-49,51,55,56}$ followed by Denmark (seven studies), ${ }^{25,31,32,35,36,38,39}$ Sweden (5 studies)$60,64,66,71,87$ and Italy (4 studies). ${ }^{74,86,90,93}$ Data from Finland, ${ }^{72,73,81}$ France, ${ }^{78,79,101}$ Norway, ${ }^{63,89,102}$ and Spain $^{62,91,98}$ were validated in three studies each. A further two studies validated EHR data from the Netherlands ${ }^{57,61}$ and one from the Czech Republic. ${ }^{92}$ All but three studies ${ }^{41,44,48}$ validated secondary care EHRs. 
Twenty-eight studies presented validation estimates for overall stroke (including both ischaemic and haemorrhagic).$25,31,32,35,38-41,44,45,48,49,56,60,63,64,66,71-73,81,86,87,91,92,98,101,102$ Ischaemic stroke was assessed in 18 studies,$25,32,38,39,47,57,62,72-74,78,79,86,90,92,93,101,102$ in all but four studies $^{62,74,79,90}$ this was done as a subgroup analysis after validating overall stroke. Similarly, haemorrhagic stroke was assessed by 21 studies; two reported results for overall haemorrhagic stroke ${ }^{32,51}$ with this the main focus of one study, ${ }^{51}$ 17 studies reported results for ICH as a subgroup analysis$25,38,39,47,51,55,57,72,73,78,86,87,89,92,93,101,102$ and 18 studies

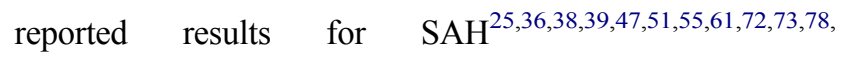
$81,86,87,89,92,93,102$ with this being the main result in two studies. $^{36,61}$

\section{Stroke Validation Results Overall}

For overall stroke, sensitivity (15 studies)$31,40,45,49,56,63,64,71,73,81,86,91,98,101,102$ was $\geq 80 \%$ (range $33-97 \%$ ) in seven studies ${ }^{49,63,64,71,73,81,102}$ and $\geq 70 \%$ in 11 studies. PPV (27 studies) $)^{25,31,32,35,38-41,45,48,49,56,60,63,64,66,71-}$ $73,81,86,87,91,92,98,101,102$ was $\geq 80 \%$ (range $20-97 \%$ ) in 19 studies. ${ }^{31,35,39-41,45,48,49,60,63,64,71,72,81,86,87,92,98}$ Nine of the studies $^{31,32,40,49,60,63,64,71,101}$ did not include codes to validate $\mathrm{SAH}$, three of which had stated this in their inclusion criteria. ${ }^{40,71,101}$ Excluding these studies did not affect the sensitivity (53-89\%) or PPV (68-97\%). Specificity and NPV, reported by five studies, were $99-100 \%{ }^{49,56,63,98}$ other than one study ${ }^{31}$ which obtained a specificity of $96 \%$ and NPV of $72 \%$.

\section{Diagnosis Type}

Three studies ${ }^{56,64,101}$ included first and recurrent overall stroke with sensitivity from $71-89 \%$ and PPV $69-81 \%$, while three studies ${ }^{32,71,73}$ also included only first stroke for which sensitivity was $85-89 \%$ and PPV $70-97 \%$.

For ischaemic stroke, the main sensitivity reported (6 studies) $)^{74,79,81,86,90,102}$ was $\geq 66 \%$ in all but one ${ }^{86}$ study

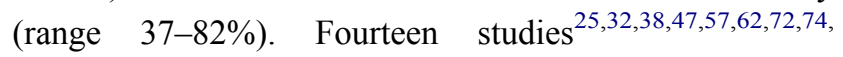
$78,79,86,90,92,102$ included one main PPV of $66-96 \%$. One study ${ }^{101}$ classified results separately for cardiac embolism, large artery atherosclerosis, lacunar infarct and ischaemic stroke of other aetiology. Sensitivity and PPV were highest in the cardiac embolism classification $(83 \%$ and $87 \%$, respectively) and lowest for other aetiology (67\% and $35 \%$, respectively). For ICH, the main sensitivity reported was 59-98\% (4 studies) ${ }^{73,86,101,102}$ and main PPV 55-96\% (15 studies). ${ }^{25,38,39,47,51,55,57,72,73,78,86,87,92,101,102}$ The sensitivity of SAH diagnoses was $35-92 \%$ (4 studies)$73,81,86,102$ and PPV was $42-96 \%$ studies). ${ }^{25,36,38,39,47,51,55,61,72,73,78,81,86,87,89,92,93,102}$

\section{Definition}

Stroke was defined in 22 of the 41 studies, 13$25,31,35,38,39,63,66,71,81,86,90,92,101,102$ used the WHO definition ${ }^{109}$

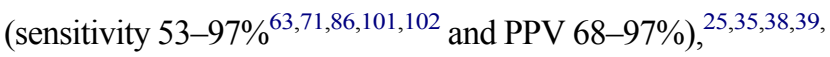
$63,66,71,81,86,92,101,102 \operatorname{seven}^{56,60,62,64,72,74,93}$ used MONICA ${ }^{110}$ (sensitivity 71-89\% ${ }^{56,64}$ and PPV 79-92\%), ${ }^{56,60,64,72}$ and two32,87 defined stroke specifically for their study (PPV 70\% and 91\%). The stroke definitions used are summarized in $\underline{\mathrm{S} 6}$ Appendix.

\section{Diagnostic Position}

For overall stroke diagnoses recorded in any diagnostic positions, sensitivity ranged from $53-97 \%{ }^{56,63,86}$ and PPV from $69-90 \% .^{25,56,63,86}$ In comparison, results only for primary position were $67-86 \%$ for sensitivity and 69-95\% for PPV. ${ }^{49,63,73,98,101}$

\section{Coding System}

Thirteen studies validated ICD-10 (PPV $20-97 \%,{ }^{31,32,38,39,45,47,55,60,63,64,71,78,92}$ sensitivity $\left.76-97 \%\right){ }^{45}$, 63,64,71,101 Four studies ${ }^{31,63,64,71}$ which excluded SAH from the stroke definition validated ICD-10 I61, I63 and I64 (sensitivity $89-97 \%$ and PPV 79-97\%). Aboa-Eboule et al ${ }^{101}$ additionally included G46 in their definition (sensitivity $77 \%$ and PPV $69 \%$ ) while Dalsgaard et $\mathrm{al}^{32}$ validated I61-I65 (PPV 70\%). In comparison, Holmqvist et $\mathrm{al}^{60}$ only included I61 and I63, and obtained PPV estimates of $92 \%$ and $89 \%$ in people with and without rheumatoid arthritis, respectively. Three studies$38,39,92$ which included SAH in the stroke definition validated I60, I61, I63 and I64 (PPV 79-86\%) and one ${ }^{45}$ additionally included I62 (PPV 96\%). The estimates for ICD-10 codes were no higher than those for ICD-8 codes (sensitivity 82\%), ${ }^{81}$ ICD-9 (PPV 20-95\%, ${ }^{40,49,66,86,91,93,98,102}$ sensitivity $33-89 \%),{ }^{40,49,86,91,98,102}$ or combinations of three ICD systems (PPV 79-97\%, ${ }^{35,72,73,87}$ sensitivity 71-85\%). ${ }^{73}$

Seven studies validated ICD-10 I63 for ischaemic stroke diagnosis (PPV 78-96\%). ${ }^{25,32,38,47,78,79,92}$ One study $^{73}$ used a broad (ICD-9433, 434, 436 and ICD-10 I63, I64) and narrow range of codes (ICD-9433, 434 and ICD-10 I63) to define ischaemic stroke, with similar sensitivity ( $82 \%$ vs $81 \%$ ) and PPV ( $84 \%$ vs $83 \%$ ). One other study ${ }^{74}$ reported results by ICD-9 codes $443 * 1$ and $434 * 1$ (PPV $86 \%$ and $90 \%$, respectively). Six studies ${ }^{25,38,55,78,89,92}$ validated ICD10 I61, with another two ${ }^{39,101}$ presumed to have also validated this code, for ICH (PPV 66-96\%) and a further three 
studies $^{86,93,102}$ validated ICD-9431 (PPV 71-78\%). For SAH, eight studies $25,38,39,47,55,78,89,92$ validated ICD-10 I60 with PPV $>90 \%$ in half of the studies (range 46-96\%), four studies $^{61,86,93,102}$ validated ICD-9430 (PPV 42-95\%), one study $^{81}$ validated ICD-8430 (PPV 85\%) and two studies ${ }^{72,87}$ validated both versions for 430 (PPV 78-79\%).

\section{Reference Standard}

In the 17 studies $25,31,32,35,38,39,45,55,56,60,63,72,79,86,87,91,92$ which used medical record review as the reference standard to validate overall stroke diagnoses, the PPV was $\geq 79 \%$ (range $20-97 \%$ ) in all but four studies. ${ }^{25,31,32,91} \mathrm{~A}$ further eight studies used a registry reference standard (PPV 88-97\%). 40,64,66,71,73,98,101,102

\section{Heterogeneity}

We were able to assess the heterogeneity between the main PPV reported in; 14 studies with 16 estimates of HF $\left(\mathrm{I}^{2}=97.0 \%\right), \quad 18$ studies with 26 estimates of MI $\left(\mathrm{I}^{2}=98.5 \%\right)$, and 19 studies with 20 estimates of stroke $\left(\mathrm{I}^{2}=97.9 \%\right)$ diagnoses. Additionally, we assessed heterogeneity between the main sensitivity for; six studies of HF $\left(\mathrm{I}^{2}=98.6 \%\right)$, four of $\mathrm{MI}\left(\mathrm{I}^{2}=74.3 \%\right)$, and 11 of stroke $\left(\mathrm{I}^{2}=98.8 \%\right)$ diagnoses. Heterogeneity between the estimates was considerable, at more than $>95 \%$ in all cases other than sensitivity estimates for MI. Furthermore, heterogeneity remained considerable after removal of studies at a high risk of bias.

\section{Overall Strength of Evidence}

GRADE showed that cross-study quality was very low for all HF outcomes (sensitivity and PPV in secondary care EHRs and PPV in primary care EHRs), low for MI sensitivity and PPV in secondary care EHRs and moderate for PPV in primary care EHRs, and very low for stroke sensitivity in secondary care EHRs and PPV in primary care EHRs and moderate for PPV in secondary care EHRs.

\section{Discussion}

\section{Summary of Findings}

Our systematic review suggests that the sensitivity of coded data in European EHRs for HF diagnoses is low at $\leq 66 \%$ in all but one study. There was also wide variation in stroke sensitivity estimates, with only half of studies $\geq 80 \%$, although three-quarters were $\geq 70 \%$. The sensitivity of ACS was higher at $\geq 80 \%$ in the vast majority of studies. The majority of studies which validated ACS diagnosis did so specifically for MI.
The PPV of all diagnoses was $\geq 80 \%$ in the majority of studies; two-thirds for HF (nearly three-quarters for secondary care EHRs), nearly three-quarters for $\mathrm{MI}$, and $70 \%$ of stroke validation studies. Where subtypes were validated, PPV was $\geq 80 \%$ for four-fifths of ischaemic stroke diagnoses but only $44 \%$ of ICH and SAH diagnoses.

The specificity and NPV were also high where available (three HF studies, three MI studies and five stroke studies). However, as most studies only included patients with the diagnosis of interest recorded in the EHR and reference standard, the results presented were mostly limited to sensitivity and PPV.

Both PPV and NPV are impacted by disease prevalence, with lower estimates for rare conditions. ${ }^{111}$ Our systematic review focused on Europe, drawing studies from 11 countries. Age-standardized prevalence of CVD in these countries is between 5000-6500 per 100,000, other than the Czech Republic ( 8700 per 100,000) which only contributed one study. ${ }^{2}$ Therefore, prevalence differences should have limited impact on our comparison of validity estimates between geographies. The prevalence of CVD increases with age, but we did not find any systematic difference in results between studies with younger or older populations.

The low sensitivity of HF diagnoses we identified is consistent with a previous systematic review validating HF diagnoses in administrative data, which identified three European studies. ${ }^{11}$ Twelve more studies have since been published and included in our review. These more recent findings, however, do not suggest any improvement in the quality of data over time. This is perhaps unsurprising given the range of clinical aetiology and presentation. The high proportion of studies we found to have a PPV of $<80 \%$ for stroke diagnoses appeared more substantial than in previous systematic reviews. ${ }^{9,12}$ We identified 15 new studies which were not included in these previous reviews. $^{25,32,45,51,56,57,61-63,74,78,89,91,92,98}$ Our results for sensitivity and PPV of MI diagnoses are consistent with previous reviews, ${ }^{8,10}$ and identified five $29,32,34,76,98$ new MI validation studies with variable results.

There was substantial heterogeneity between the sensitivity and PPV estimates for all three acute CVD diagnoses. Heterogeneity was likely because studies differed in multiple ways; for example, even among studies which used medical record review as the reference standard, differences in study time period impacted upon the ICD version used. The heterogeneity caused by variable methods was highlighted in previous systematic reviews of 
atrial fibrillation and dementia diagnoses recorded in routine health data. ${ }^{112,113}$

\section{Defining Diagnosis in the EHR}

We were most interested in the results of ICD-10 validation, as this is the latest ICD coding system which is widely used in Europe and elsewhere. In McCormick et al's ${ }^{10}$ review of MI diagnoses in administrative data, the authors noted a lack of ICD-10 validation with only three studies identified, whereas our review identified 10 . Nevertheless, even within ICD-10, combinations of codes used, and therefore their validity, differed, which highlights the importance of tailoring codes to each research question. Codes are arguably even more important when using other, more complex coding systems such as Read codes, which are used in UK primary care data and can generate vast numbers of codes for every clinical condition.

\section{Defining Diagnosis in the Reference Standard}

There is no single recommended gold standard to determine the validity of EHR data. ${ }^{114}$ Nearly three-quarters (74\%) of studies used medical records; more frequently for HF diagnoses $(85 \%)$ than ACS $(71 \%)$ or stroke (68\%). This difference may be due to availability of MI and stroke registries, used in $26 \%$ and $22 \%$ of studies, respectively. No differences in the performance of the reference standard methods were discernable, probably due to heterogeneity.

Criteria to define CVD, especially MI, have been refined over time, driven by the development of more sensitive and specific biomarkers, and more precise imaging techniques. ${ }^{100}$ However, we did not identify any temporal trends in the accuracy of MI recording, again likely due to overall study heterogeneity.

When validating HF, which can vary in clinical aetiology and presentation, clarity on the criteria used to define, with explicit classification of acute and chronic HF along with ejection fraction would benefit understanding of results.

\section{Comparing and Combining Data Sources}

Only $14(17 \%)$ studies validated primary care systems, more than half of which were in the UK. Using primary care EHRs may be beneficial for research into conditions such as HF which are frequently managed in primary care; in our study, $30 \%$ of HF EHR validation studies used primary care data, compared to $16 \%$ for ACS and $7 \%$ for stroke studies. For acute severe conditions resulting in hospitalization, secondary care records should be the most reliable data source. Where possible, the use of linked data to increase the ascertainment of acute CVD events should be considered.

\section{Implications for Future Research}

EHR-based research is a growing field - widely used in observational analyses and increasingly employed in trials. ${ }^{115}$ Researchers should consider the level of validity necessary for their own CVD outcome definition. When a composite outcome, such as MACE, is used researchers may need to address differing sensitivity in the individual components of the outcome. In studies which investigate CVD incidence, a sensitive definition is particularly important. For example, EHR data are being used for rapid COVID-19 pandemic analyses such as; the impact the virus has in those with CVD, CVD as an outcome after infection with the virus, and excess death estimates. ${ }^{116}$ It is important that these rapid analyses consider the validity of the data and definitions used. Conversely, in a pragmatic trial recruitment, a specific definition is likely more important than a sensitive one.

\section{Strengths and Limitations}

Our systematic review provides a comprehensive and up-todate evaluation of the validity of acute CVD diagnoses in European EHRs, conducted without language or time restrictions using a broad search strategy. Two independent reviewers performed our study selection, and native speaking collaborators translated foreign language articles. Similar to other systematic reviews of validation studies, we repurposed the QUADAS-2 risk of bias tool developed for diagnostic test accuracy. Additionally, we followed the diagnostic test accuracy GRADE methodology to assess the overall evidence base.

Our work is not without limitations. Firstly, only one reviewer completed full data extraction and risk of bias assessment due to resource constraints, although a sample of $20 \%$ of studies had data dual extracted. Secondly, we limited our study to Europe, so theoretically our results are only generalizable to European countries. All previous systematic reviews $^{8-12}$ on the validity of acute CVD diagnoses included both EHRs and claim-based systems, while most studies included in each of these reviews were from North America. From these existing reviews, it was unclear if the validity of EHRs differed to claims-based datasets, which reflect payments related to medical care given. Despite this, we obtained similar results to the 
previous reviews. Thirdly, our review focused on acute CVD events so excluded results from studies that validated broader diagnoses of ischaemic heart disease or cerebrovascular disease, which again limits generalizability to these specific conditions.

\section{Recommendations}

For ACS and stroke diagnoses, most sensitivity and PPV results were reasonably high, providing confidence in the use of European EHR data for research into these conditions. However, there was considerable heterogeneity between studies. Sensitivity for HF diagnoses was low, and our GRADE assessment found very low quality for all HF outcomes. For studies of HF, we strongly recommend either validating the definition or referring to existing validation studies to develop the case definition. New validation studies of HF diagnoses should report whether the diagnoses validated are for acute or chronic presentation and HF with reduced ejection fraction or preserved ejection fraction. These principles are also applicable to future ACS and stroke validation studies. Identifying specific stroke subtypes can be difficult; analysis of all stroke subtypes combined is preferable.

\section{Conclusions}

Our review on the accuracy of HF, ACS and stroke diagnoses in European EHRs should guide researchers in their selection of data sources and CVD definitions for epidemiological studies. Generally, the data assessed was of reasonable quality. However, it is difficult to summarize validity given the heterogeneity between studies. Where possible, researchers should validate data before use or carefully interpret the results of previous validation studies to consider the impact validity has on research findings. Additionally, the use of linked data will bolster quality.

\section{Acknowledgments}

We thank Hanne-Dorthe Emborg and Elisabeth Bondesson for their translations of Danish and Swedish language articles.

\section{Author Contributions}

All authors made a significant contribution to the work reported, whether that is in the conception, study design, execution, acquisition of data, analysis and interpretation, or in all these areas; took part in drafting, revising or critically reviewing the article; gave final approval of the version to be published; have agreed on the journal to which the article has been submitted; and agree to be accountable for all aspects of the work.

\section{Funding}

J.A.D. is funded by a British Heart Foundation NonClinical PhD Studentship (FS/18/71/33938) and C.W.G is funded by a Wellcome Intermediate Clinical Fellowship (201440/Z/16/Z). The funders had no role in study design, data collection and analysis, preparation of the manuscript, or the decision to publish.

\section{Disclosure}

Charlotte Warren-Gash reports grants from Wellcome, grants from British Heart Foundation, during the conduct of the study; personal fees from Sanofi Pasteur, outside the submitted work. The authors declare no other potential conflicts of interest for this work.

\section{References}

1. World Health Organization. The top 10 causes of death. https:// www.who.int/en/news-room/fact-sheets/detail/the-top-10-causesof-death. Accessed February 11, 2019.

2. European Heart Network. CVD statistics 2017; 2017. Available from: http://www.ehnheart.org/cvd-statistics/cvd-statistics-2017. html. Accessed October 9, 2019 ..

3. Denaxas SC, Morley KI. Big biomedical data and cardiovascular disease research: opportunities and challenges. Eur Hear J - Qual Care Clin Outcomes. 2015;1(1):9-16. doi:10.1093/ehjqcco/ qcv005

4. Herrett E, Thomas SL, Schoonen WM, Smeeth L, Hall AJ. Validation and validity of diagnoses in the general practice research database: a systematic review. $\mathrm{Br} J$ Clin Pharmacol. 2010;69(1):4-14. doi:10.1111/j.1365-2125.2009.03537.x

5. Sund R. Quality of the Finnish hospital discharge register: a systematic review. Scand J Public Health. 2012;40(6):505-515. doi:10.1177/1403494812456637

6. Ludvigsson JF, Andersson E, Ekbom A, et al. External review and validation of the Swedish national inpatient register. BMC Public Health. 2011;11:450. doi:10.1186/1471-2458-11-450

7. Schmidt M, Schmidt SAJ, Sandegaard JL, Ehrenstein V, Pedersen L, Sørensen HT. The Danish National Patient Registry: a review of content, data quality, and research potential. Clin Epidemiol. 2015;7:449-490. doi:10.2147/CLEP.S91125

8. Rubbo B, Fitzpatrick NK, Denaxas S, et al. Use of electronic health records to ascertain, validate and phenotype acute myocardial infarction: a systematic review and recommendations. Int $J$ Cardiol. 2015;187:705-711. doi:10.1016/j.ijcard.2015.03.075

9. Woodfield R, Grant I, Sudlow CLM; UK Biobank Follow-Up and Outcomes Working Group. Accuracy of electronic health record data for identifying stroke cases in large-scale epidemiological studies: a systematic review from the UK biobank stroke outcomes group. PLoS One. 2015;10(10):e0140533. doi:10.1371/ journal.pone. 0140533

10. McCormick N, Lacaille D, Bhole V, Avina-Zubieta JA, Guo Y. Validity of myocardial infarction diagnoses in administrative databases: a systematic review. PLoS One. 2014;9(3):e92286. doi:10.1371/journal.pone.0092286 
11. McCormick N, Lacaille D, Bhole V, Avina-Zubieta JA. Validity of heart failure diagnoses in administrative databases: a systematic review and meta-analysis. PLoS One. 2014;9(8):e104519. doi:10.1371/journal.pone.0104519

12. McCormick N, Bhole V, Lacaille D, Avina-Zubieta JA. Validity of diagnostic codes for acute stroke in administrative databases: a systematic review. PLoS One. 2015;10(8):e0135834. doi:10.1371/ journal.pone.0135834

13. Poudel I, Tejpal C, Rashid H, Jahan N. Major adverse cardiovascular events: an inevitable outcome of ST-elevation myocardial infarction? A literature review. Cureus. 2019;11(7). doi:10.7759/ cureus. 5280

14. Davidson JA, Banerjee A, Muzambi R, Smeeth L, Warren-Gash C. Validity of acute cardiovascular outcome diagnoses in European electronic health records: a systematic review protocol. BMJ Open. 2019;9(10). doi:10.1136/bmjopen-2019-031373

15. Ponikowski P, Voors AA, Anker SD, et al. 2016 ESC Guidelines for the diagnosis and treatment of acute and chronic heart failure: the task force for the diagnosis and treatment of acute and chronic heart failure of the European Society of Cardiology (ESC) developed with the special contribution. Eur Heart J. 2016;37 (27):2129-2200.

16. Luepker RV, Apple FS, Christenson RH, et al. Case definitions for acute coronary heart disease in epidemiology and clinical research studies. Circulation. 2003;108(20):2543-2549. doi:10.1161/01. CIR.0000100560.46946.EA

17. Sacco RL, Kasner SE, Broderick JP, et al. An updated definition of stroke for the 21 st century: a statement for healthcare professionals from the American Heart Association/American Stroke Association. Stroke. 2013;44(7):2064-2089. doi:10.1161/ STR.0b013e318296aeca

18. Whiting PF, Rutjes AWS, Westwood ME, et al. QUADAS-2: a revised tool for the quality assessment of diagnostic accuracy studies. Ann Intern Med. 2011;155(8):529. doi:10.7326/00034819-155-8-201110180-00009

19. The Cochrane Collaboration. Cochrane handbook for systematic reviews of interventions. Version 5. (Higgins JP, Green S, eds.); 2011. Available from: https://handbook-5-1.cochrane.org/. Accessed September 9, 2020.

20. Nyaga VN, Arbyn M, Aerts M. Metaprop: a Stata command to perform meta-analysis of binomial data. Arch Public Health. 2014;72(1):1-10. doi:10.1186/2049-3258-72-39

21. Schünemann HJ, Oxman AD, Brozek J, et al. Grading quality of evidence and strength of recommendations for diagnostic tests and strategies. BMJ. 2008;336:1106-1110. doi:10.1136/ bmj.39500.677199.ae

22. Bork CS, Al-Zuhairi KS, Hansen SM, Delekta J, Joensen AM. Accuracy of angina pectoris and acute coronary syndrome in the Danish National Patient Register. Dan Med J. 2017;64(5).

23. Coloma PM, Valkhoff VE, Mazzaglia G, et al. Identification of acute myocardial infarction from electronic healthcare records using different disease coding systems: a validation study in three European countries. BMJ Open. 2013;3(6):e002862. doi:10.1136/bmjopen-2013-002862

24. Kümler T, Gislason GH, Kirk V, et al. Accuracy of a heart failure diagnosis in administrative registers. Eur J Heart Fail. 2008;10 (7):658-660. doi:10.1016/j.ejheart.2008.05.006

25. Lühdorf P, Overvad K, Schmidt EB, Johnsen SP, Bach FW. Predictive value of stroke discharge diagnoses in the Danish National Patient Register. Scand J Public Health. 2017;45 (6):630-636. doi:10.1177/1403494817716582

26. Madsen M, Balling H, Eriksen LS. [The validity of the diagnosis of acute myocardial infarction in 2 registries: the Heart Registry compared to the National Patient Registry]. Ugeskr Laeger. 1990;152(5):308-314. Danish.
27. Madsen M, Davidsen M, Rasmussen S, Abildstrom SZ, Osler M. The validity of the diagnosis of acute myocardial infarction in routine statistics: a comparison of mortality and hospital discharge data with the Danish MONICA registry. J Clin Epidemiol. 2003;56 (2):124-130. doi:10.1016/S0895-4356(02)00591-7

28. Mard S, Nielsen FE. Positive predictive value and impact of misdiagnosis of a heart failure diagnosis in administrative registers among patients admitted to a university hospital cardiac care unit. Clin Epidemiol. 2010;2:235-239. doi:10.2147/CLEP.S12457

29. Sundbøll J, Adelborg K, Munch T, et al. Positive predictive value of cardiovascular diagnoses in the Danish National Patient Registry: a validation study. BMJ Open. 2016;6(11):e012832. doi:10.1136/bmjopen-2016-012832

30. Thygesen SK, Christiansen CF, Christensen S, Lash TL, Sørensen HT. The predictive value of ICD-10 diagnostic coding used to assess Charlson comorbidity index conditions in the populationbased Danish National Registry of Patients. BMC Med Res Methodol. 2011;11:83. doi:10.1186/1471-2288-11-83

31. Wildenschild C, Mehnert FW, Thomsen R, et al. Registration of acute stroke: validity in the Danish Stroke Registry and the Danish National Registry of Patients. Clin Epidemiol. 2013;6:27. doi:10.2147/CLEP.S50449

32. Dalsgaard E-M, Witte DR, Charles M, Jørgensen ME, Lauritzen T, Sandbæk A. Validity of Danish register diagnoses of myocardial infarction and stroke against experts in people with screendetected diabetes. BMC Public Health. 2019;19(1):228. doi:10.1186/s12889-019-6549-z

33. Delekta J, Hansen S, AlZuhairi K, Bork C, Joensen A. The validity of the diagnosis of heart failure (I50.0-I50.9) in the Danish National Patient Register. Dan Med J. 2018;65(4): A5470.

34. Egholm G, Madsen M, Thim T, et al. Evaluation of algorithms for registry-based detection of acute myocardial infarction following percutaneous coronary intervention. Clin Epidemiol. 2016;8:415423. doi:10.2147/CLEP.S108906

35. Frost L, Andersen LV, Vestergaard P, Husted S, Mortensen LS. Trend in mortality after stroke with atrial fibrillation. Am J Med. 2007;120(1):47-53. doi:10.1016/j.amjmed.2005.12.027

36. Gaist D. Risk of subarachnoid haemorrhage in first degree relatives of patients with subarachnoid haemorrhage: follow up study based on national registries in Denmark. BMJ. 2000;320 (7228):141-145. doi:10.1136/bmj.320.7228.141

37. Joensen AM, Jensen MK, Overvad K, et al. Predictive values of acute coronary syndrome discharge diagnoses differed in the Danish National Patient Registry. J Clin Epidemiol. 2009;62 (2):188-194. doi:10.1016/J.JCLINEPI.2008.03.005

38. Johnsen SP, Overvad K, Sørensen HT, Tjønneland A, Husted SE. Predictive value of stroke and transient ischemic attack discharge diagnoses in The Danish National Registry of Patients. J Clin Epidemiol. 2002;55(6):602-607. doi:10.1016/S0895-4356(02) 00391-8

39. Krarup L-H, Boysen G, Janjua H, Prescott E, Truelsen T. Validity of stroke diagnoses in a National Register of Patients. Neuroepidemiology. 2007;28(3):150-154. doi:10.1159/000102143

40. Barer D, Ellul J, Watkins C. Correcting outcome data for case mix in stroke medicine. BMJ. 1996;313(7063):1005-1006. doi:10.1136/bmj.313.7063.1005c

41. Cook M, Baker N, Lanes S, Bullock R, Wentworth C, Michael Arrighi H. Incidence of stroke and seizure in Alzheimer's disease dementia. Age Ageing. 2015;44(4):695-699. doi:10.1093/ageing/ afv061

42. McAlpine R, Pringle S, Pringle T, Lorimer R, MacDonald TM. A study to determine the sensitivity and specificity of hospital discharge diagnosis data used in the MICA study. Pharmacoepidemiol Drug Saf. 1998;7(5):311-318. doi:10.1002/ (SICI)1099-1557(199809/10)7:5<311::AID-PDS371>3.0.CO;2-O 
43. Pfister R, Michels G, Wilfred J, Luben R, Wareham NJ, Khaw KT. Does ICD-10 hospital discharge code I50 identify people with heart failure? A validation study within the EPIC-Norfolk study. Int $J$ Cardiol. 2013;168(4):4413-4414. doi:10.1016/j. ijcard.2013.05.031

44. Ruigómez A, Martín-Merino E, Rodríguez LAG. Validation of ischemic cerebrovascular diagnoses in the health improvement network (THIN). Pharmacoepidemiol Drug Saf. 2010;19 (6):579-585. doi:10.1002/PDS.1919

45. Sansom LT, Ramadan H. Stroke incidence: sensitivity of hospital data coding of acute stroke. Int J Stroke. 2015;10(6):E70. doi:10.1111/ijs. 12577

46. Van Staa TP, Abenhaim L. The quality of information recorded on a UK database of primary care records: a study of hospitalizations due to hypoglycemia and other conditions. Pharmacoepidemiol Drug Saf. 1994;3(1):15-21. doi:10.1002/pds.2630030106

47. Wright FL, Green J, Canoy D, Cairns BJ, Balkwill A, Beral V. Vascular disease in women: comparison of diagnoses in hospital episode statistics and general practice records in England. $B M C$ Med Res Methodol. 2012;12(1):161. doi:10.1186/1471-2288-12161

48. Zhou EH, Gelperin K, Levenson MS, Rose M, Hsueh YH, Graham DJ. Risk of acute myocardial infarction, stroke, or death in patients initiating olmesartan or other angiotensin receptor blockers - a cohort study using the clinical practice research datalink. Pharmacoepidemiol Drug Saf. 2014;23(4):340-347. doi:10.1002/pds.3549

49. Davenport RJ, Dennis MS, Warlow CP. The accuracy of Scottish Morbidity Record (SMR1) data for identifying hospitalised stroke patients. Health Bull (Raleigh). 1996;54(5):402-405.

50. Donnan PT, Dougall HT, Sullivan FM. Optimal strategies for identifying patients with myocardial infarction in general practice. Fam Pract. 2003;20(6):706-710. doi:10.1093/fampra/ cmg614

51. Gaist D, Wallander M-A, González-Pérez A, García-Rodríguez LA. Incidence of hemorrhagic stroke in the general population: validation of data from The Health Improvement Network. Pharmacoepidemiol Drug Saf. 2013;22(2):176-182. doi:10.10 02/pds.3391

52. Hammad TA, McAdams MA, Feight A, Iyasu S, Dal Pan GJ. Determining the predictive value of Read/OXMIS codes to identify incident acute myocardial infarction in the General Practice Research Database. Pharmacoepidemiol Drug Saf. 2008;17 (12):1197-1201. doi:10.1002/pds.1672

53. Herrett E, Shah AD, Boggon R, et al. Completeness and diagnostic validity of recording acute myocardial infarction events in primary care, hospital care, disease registry, and national mortality records: cohort study. BMJ. 2013;346(may203):f2350-f2350. doi:10.1136/bmj.f2350

54. Khand AU, Shaw M, Gemmel I, Cleland JGF. Do discharge codes underestimate hospitalisation due to heart failure? Validation study of hospital discharge coding for heart failure. Eur $J$ Heart Fail. 2005;7(5):792-797. doi:10.1016/j. ejheart.2005.04.001

55. Kirkman MA, Mahattanakul W, Gregson BA, Mendelow AD. The accuracy of hospital discharge coding for hemorrhagic stroke. Acta Neurol Belg. 2009;109(2):114-119.

56. Kivimäki M, Batty GD, Singh-Manoux A, Britton A, Brunner EJ, Shipley MJ. Validity of cardiovascular disease event ascertainment using linkage to UK hospital records. Epidemiology. 2017;28(5):735-739. doi:10.1097/EDE.0000000000000688

57. Ekker MS, Verhoeven JI, Vaartjes I, van Nieuwenhuizen KM, Klijn CJM, de Leeuw FE. Stroke incidence in young adults according to age, subtype, sex, and time trends. Neurology. 2019;92(21):e2444-e2454. doi:10.1212/WNL.000000000000 7533
58. Hammar N, Alfredsson L, Rosén M, Spetz CL, Kahan T, Ysberg AS. A national record linkage to study acute myocardial infarction incidence and case fatality in Sweden. Int $J$ Epidemiol. 2001;30(Suppl 1):S30-4. doi:10.1093/ije/30.supp1_1.S30

59. Heerdink ER, Leufkens HG, Herings RM, Ottervanger JP, Stricker BH, Bakker A. NSAIDs associated with increased risk of congestive heart failure in elderly patients taking diuretics. Arch Intern Med. 1998;158(10):1108-1112. doi:10.1001/ archinte.158.10.1108

60. Holmqvist M, Gränsmark E, Mantel Ä, et al. Occurrence and relative risk of stroke in incident and prevalent contemporary rheumatoid arthritis. Ann Rheum Dis. 2013;72(4):541-546. doi:10.1136/annrheumdis-2012-201387

61. Nieuwkamp DJ, Vaartjes I, Algra A, Rinkel GJE, Bots ML. Risk of cardiovascular events and death in the life after aneurysmal subarachnoid haemorrhage: a nationwide study. Int J Stroke. 2014;9(8):1090-1096. doi:10.1111/j.1747-4949.2012.00875.x

62. Vila-Corcoles A, Satue-Gracia E, Ochoa-Gondar O, et al. Incidence and lethality of ischaemic stroke among people 60 years or older in the region of Tarragona (Spain), 2008-2011. Rev Neurol. 2014;59(11):490-496.

63. Varmdal T, Bakken IJ, Janszky I, et al. Comparison of the validity of stroke diagnoses in a medical quality register and an administrative health register. Scand J Public Health. 2016;44(2):143149. doi:10.1177/1403494815621641

64. Köster M, Asplund K, Johansson Å, Stegmayr B. Refinement of Swedish Administrative Registers to monitor stroke events on the national level. Neuroepidemiology. 2013;40(4):240-246. doi: $10.1159 / 000345953$

65. Merry AHH, Boer JMA, Schouten LJ, et al. Validity of coronary heart diseases and heart failure based on hospital discharge and mortality data in the Netherlands using the cardiovascular registry Maastricht cohort study. Eur J Epidemiol. 2009;24(5):237-247. doi:10.1007/s10654-009-9335-x

66. Stegmayr B, Asplund K. Measuring stroke in the population: quality of routine statistics in comparison with a populationbased stroke registry. Neuroepidemiology. 1992;11(4-6):204213. doi: $10.1159 / 000110933$

67. Mähönen M, Jula A, Harald $\mathrm{K}$, et al. The validity of heart failure diagnoses obtained from administrative registers. Eur J Prev Cardiol. 2013;20(2):254-259. doi:10.1177/ 2047487312438979

68. Pajunen P, Koukkunen H, Ketonen M, et al. The validity of the Finnish Hospital Discharge Register and causes of death register data on coronary heart disease. Eur J Cardiovasc Prev Rehabil. 2005;12(2):132-137.

69. Pietilä K, Tenkanen L, Mänttäri M, Manninen V. How to define coronary heart disease in register-based follow-up studies: experience from the Helsinki Heart Study. Ann Med. 1997;29(3):253259. doi:10.3109/07853899708999343

70. Rapola JM, Virtamo J, Korhonen P, et al. Validity of diagnoses of major coronary events in national registers of hospital diagnoses and deaths in Finland. Eur J Epidemiol. 1997;13(2):133-138. doi:10.1023/A:1007380408729

71. Appelros P, Terént A. Validation of the Swedish inpatient and cause-of-death registers in the context of stroke. Acta Neurol Scand. 2011;123(4):289-293. doi:10.1111/j.1600-0404.2010. 01402.x

72. Leppälä JM, Virtamo J, Heinonen OP. Validation of stroke diagnosis in the National Hospital Discharge Register and the Register of Causes of Death in Finland. Eur J Epidemiol. 1999;15(2):155160. doi:10.1023/A:1007504310431

73. Tolonen H, Salomaa V, Torppa J, et al. The validation of the Finnish Hospital Discharge Register and causes of death register data on stroke diagnoses. Eur J Cardiovasc Prev Rehabil. 2007;14(3):380-385. doi:10.1097/01.hjr.0000239466.26132.f2 
74. Baldereschi M, Balzi D, Di Fabrizio V, et al. Administrative data underestimate acute ischemic stroke events and thrombolysis treatments: data from a multicenter validation survey in Italy. PLoS One. 2018;13(3):e0193776. doi:10.1371/journal.pone.0193776

75. Barchielli A, Balzi D, Naldoni P, et al. Hospital discharge data for assessing myocardial infarction events and trends, and effects of diagnosis validation according to MONICA and AHA criteria. $J$ Epidemiol Community Health. 2012;66(5):462-467. doi:10.1136/ jech.2010.110908

76. Bezin J, Girodet P-O, Rambelomanana S, et al. Choice of ICD-10 codes for the identification of acute coronary syndrome in the French hospitalization database. Fundam Clin Pharmacol. 2015;29(6):586-591. doi:10.1111/fcp.12143

77. Bosco-Lévy P, Duret S, Picard F, et al. Diagnostic accuracy of the International Classification of Diseases, Tenth Revision, codes of heart failure in an administrative database. Pharmacoepidemiol Drug Saf. 2019;28(2):194-200. doi:10.1002/pds.4690

78. Giroud M, Hommel M, Benzenine E, Fauconnier J, Béjot Y, Quantin C. Positive predictive value of French hospitalization discharge codes for stroke and transient ischemic attack. Eur Neurol. 2015;74:92-99. doi:10.1159/000438859

79. Haesebaert J, Termoz A, Polazzi S, et al. Can hospital discharge databases be used to follow ischemic stroke incidence? Stroke. 2013;44(7):1770-1774. doi:10.1161/STROKEAHA.113.001300

80. Hammar N, Larsen FF, de Faire U. Are geographical differences and time trends in myocardial infarction incidence in Sweden real? Validity of hospital discharge diagnoses. J Clin Epidemiol. 1994;47(6):685-693. doi:10.1016/0895-4356(94)90216-x

81. Heliövaara M, Reunanen A, Aromaa A, Knekt P, Aho K, Suhonen O. Validity of hospital discharge data in a prospective epidemiological study on stroke and myocardial infarction. Acta Med Scand. 1984;216(3):309-315. doi:10.1111/j.0954-6820.1984.tb03809.x

82. Hjerpe P, Merlo J, Ohlsson H, Bengtsson Boström K, Lindblad U. Validity of registration of ICD codes and prescriptions in a research database in Swedish primary care: a cross-sectional study in Skaraborg primary care database. BMC Med Inform Decis Mak. 2010;10(1). doi:10.1186/1472-6947-10-23

83. Ingelsson E, Ärnlöv J, Sundström J, Lind L. The validity of a diagnosis of heart failure in a hospital discharge register. Eur $J$ Heart Fail. 2005;7(5):787-791. doi:10.1016/j.ejheart.2004.12.007

84. Joensuu T, Näyhä S. Reliability of hospital discharge diagnoses of acute myocardial infarction. Scand J Soc Med. 1992;20(2):85-86. doi:10.1177/140349489202000204

85. Kaspar M, Fette G, Güder G, et al. Underestimated prevalence of heart failure in hospital inpatients: a comparison of ICD codes and discharge letter information. Clin Res Cardiol. 2018;107 (9):778-787. doi:10.1007/s00392-018-1245-z

86. Leone MA, Capponi A, Varrasi C, Tarletti R, Monaco F. Accuracy of the ICD-9 codes for identifying TIA and stroke in an Italian automated database. Neurol Sci. 2004;25(5):281-288. doi:10.1007/s10072-004-0355-8

87. Lindblad U, Råstam L, Ranstam J, Peterson M. Validity of register data on acute myocardial infarction and acute stroke: the Skaraborg Hypertension Project. Scand J Soc Med. 1993;21 (1):3-9. doi:10.1177/140349489302100102

88. Nilsson AC, Spetz CL, Carsjö K, Nightingale R, Smedby B. [Reliability of the hospital registry. The diagnostic data are better than their reputation]. Lakartidningen. 1994;91(7):598,603-605. Swedish.

89. Øie LR, Madsbu MA, Giannadakis C, et al. Validation of intracranial hemorrhage in the Norwegian Patient Registry. Brain Behav. 2018;8(2):e00900. doi:10.1002/brb3.900

90. Rinaldi R, Vignatelli L, Galeotti M, Azzimondi G, de Carolis P. Accuracy of ICD-9 codes in identifying ischemic stroke in the General Hospital of Lugo di Romagna (Italy). Neurol Sci. 2003;24(2):65-69. doi:10.1007/s100720300074
91. Rodrigo-Rincon I, Martin-Vizcaino MP, Tirapu-Leon B, ZabalzaLopez P, Abad-Vicente FJ, Merino-Peralta A. Validity of the clinical and administrative databases in detecting post-operative adverse events. Int J Qual Health Care. 2015;27(4):267-275. doi:10.1093/intqhe/mzv039

92. Sedova P, Brown RD, Zvolsky M, et al. Validation of stroke diagnosis in the National Registry of Hospitalized Patients in the Czech Republic. J Stroke Cerebrovasc Dis. 2015;24 (9):2032-2038. doi:10.1016/j.jstrokecerebrovasdis.2015.04.019

93. Spolaore P, Brocco S, Fedeli U, et al. Measuring accuracy of discharge diagnoses for a region-wide surveillance of hospitalized strokes. Stroke. 2005;36(5):1031-1034. doi:10.1161/01.STR.000 $0160755.94884 .4 \mathrm{a}$

94. Valk MJ, Mosterd A, Broekhuizen BDL, et al. Overdiagnosis of heart failure in primary care: a cross-sectional study. $\mathrm{Br} \mathrm{J}$ Gen Pract. 2016;66(649):e587-e592. doi:10.3399/bjgp16X685705

95. van Doorn S, Brakenhoff TB, Moons KGM, et al. The effects of misclassification in routine healthcare databases on the accuracy of prognostic prediction models: a case study of the CHA2DS2VASc score in atrial fibrillation. Diagnostic Progn Res. 2017;1(1). doi:10.1186/s41512-017-0018-x

96. Verdú-Rotellar JM, Frigola-Capell E, Alvarez-Pérez R, et al. Validation of heart failure diagnosis registered in primary care records in two primary care centres in Barcelona (Spain) and factors related. A cross-sectional study. Eur J Gen Pract. 2017;23(1):107-113. doi:10.1080/13814788.2017.1305104

97. Gini R, Schuemie MJ, Mazzaglia G, et al. Automatic identification of type 2 diabetes, hypertension, ischaemic heart disease, heart failure and their levels of severity from Italian General Practitioners' electronic medical records: a validation study. BMJ Open. 2016;6(12):e012413. doi:10.1136/bmjopen-2016012413

98. Bernal JL, Barrabés JA, Íñiguez A, et al. Clinical and administrative data on the research of acute coronary syndrome in Spain. Minimum basic data set validity. Rev Española Cardiol (English Ed). 2019;72(1):56-62. doi:10.1016/j.rec.2018.01.026

99. Mähönen M, Salomaa V, Brommels M, et al. The validity of hospital discharge register data on coronary heart disease in Finland. Eur J Epidemiol. 1997;13(4):403-415. doi:10.1023/ A:1007306110822

100. Palomäki P, Miettinen H, Mustaniemi H, et al. Diagnosis of acute myocardial infarction by MONICA and FINMONICA diagnostic criteria in comparison with hospital discharge diagnosis. J Clin Epidemiol. 1994;47(6):659-666. doi:10.1016/0895-4356(94) 90213-5

101. Aboa-Eboulé C, Mengue D, Benzenine E, et al. How accurate is the reporting of stroke in hospital discharge data? A pilot validation study using a population-based stroke registry as control. J Neurol. 2013;260(2):605-613. doi:10.1007/s00415012-6686-0

102. Ellekjaer H, Holmen J, Krüger O, Terent A. Identification of incident stroke in Norway: hospital discharge data compared with a population-based stroke register. Stroke. 1999;30(1):5660. doi:10.1161/01.STR.30.1.56

103. Ho KKL, Pinsky JL, Kannel WB, Levy D. The epidemiology of heart failure: the Framingham Study. J Am Coll Cardiol. 1993;22 (SUPPL. 4):6-43. doi:10.1016/0735-1097(93)90455-A

104. Carlson KJ, Lee DCS, Goroll AH, Leahy M, Johnson RA. An analysis of physicians' reasons for prescribing long-term digitalis therapy in outpatients. J Chronic Dis. 1985;38(9):733-739. doi:10.1016/0021-9681(85)90115-8

105. Yancy CW, Jessup M, Bozkurt B, et al. 2013 ACCF/AHA guideline for the management of heart failure: a report of the American College Of Cardiology Foundation/American Heart Association Task Force On Practice Guidelines. Circulation. 2013;128(16). doi:10.1161/CIR.0b013e31829e8776 
106. Tunstall-Pedoe H, Kuulasmaa K, Amouyel P, Arveiler D, Rajakangas AM, Pajak A. Myocardial infarction and coronary deaths in the World Health Organization MONICA project: registration procedures, event rates, and case-fatality rates in 38 populations from 21 countries in four continents. Circulation. 1994;90 (1):583-612. doi:10.1161/01.cir.90.1.583

107. Thygesen K, Alpert JS, White HD. Universal definition of myocardial infarction. Circulation. 2007;116(22):2634-2653. doi:10.1161/CIRCULATIONAHA.107.187397

108. Thygesen K, Alpert JS, Jaffe AS, Simoons ML, Chaitman BR, White HD. Third universal definition of myocardial infarction. Circulation. 2012;126(16):2020-2035. doi:10.1161/CIR.0b013e3 $1826 \mathrm{e} 1058$

109. Aho K, Harmsen P, Hatano S, Marquardsen J, Smirnov VE, Strasser T. Cerebrovascular disease in the community: results of a WHO collaborative study. Bull World Health Organ. 1980;58 (1):113-130.

110. Thorvaldsen P, Kuulasmaa K, Rajakangas A-M, Rastenyte D, Sarti C, Wilhelmsen L. Stroke trends in the WHO MONICA project. Stroke. 1997;28(3):500-506. doi:10.1161/01.STR.28. 3.500

111. Brenner H, Gefeller O. Variation of sensitivity, specificity, likelihood ratios and predictive values with disease prevalence. Stat Med. 1997;16(9):981-991. doi:10.1002/(SICI)1097-0258(199 70515)16:9<981::AID-SIM510>3.0.CO;2-N
112. Yao RJR, Andrade JG, Deyell MW, Jackson H, McAlister FA, Hawkins NM. Sensitivity, specificity, positive and negative predictive values of identifying atrial fibrillation using administrative data: a systematic review and meta-analysis. Clin Epidemiol. 2019;11:753-767. doi:10.2147/CLEP.S206267

113. McGuinness LA, Warren-Gash C, Moorhouse LR, Thomas SL. The validity of dementia diagnoses in routinely collected electronic health records in the United Kingdom: a systematic review. Pharmacoepidemiol Drug Saf. 2019;28(2):244-255. doi:10.1002/ pds. 4669

114. Nissen F, Quint JK, Morales DR, Douglas IJ. How to validate a diagnosis recorded in electronic health records. Breathe. 2019;15 (1):64-68. doi:10.1183/20734735.0344-2018

115. Hemingway H, Asselbergs FW, Danesh J, et al. Big data from electronic health records for early and late translational cardiovascular research: challenges and potential. Eur Heart J. 2018;39 (16):1481-1495. doi:10.1093/eurheartj/ehx487

116. Banerjee A, Pasea L, Harris S, et al. Estimating excess 1-year mortality from COVID-19 according to underlying conditions and age in England: a rapid analysis using NHS health records in 3.8 million adults. medRxiv. 2020. doi:10.1101/2020.03.22.20040287

\section{Publish your work in this journal}

Clinical Epidemiology is an international, peer-reviewed, open access, online journal focusing on disease and drug epidemiology, identification of risk factors and screening procedures to develop optimal preventative initiatives and programs. Specific topics include: diagnosis, prognosis, treatment, screening, prevention, risk factor modification,

Submit your manuscript here: https://www.dovepress.com/clinical-epidemiology-journal systematic reviews, risk \& safety of medical interventions, epidemiology \& biostatistical methods, and evaluation of guidelines, translational medicine, health policies \& economic evaluations. The manuscript management system is completely online and includes a very quick and fair peer-review system, which is all easy to use. 